\title{
1 Peaceful behaviour: a strategy employed by an obligate nest invader 2 to avoid conflict with its host species
}

3

\section{Abstract}

15 In addition to its builders, termite nests are known to house a variety of secondary,

16 opportunistic termite species, but little is known about the mechanisms governing the

17 maintenance of such associations. In a single nest, host and intruder are likely to engage in

18 intense conflict, due to their nestmate discrimination system. An intriguing question is how

19 individuals cope with such a burden in the long term. Evasive behaviour has been previously

20 suggested as a mechanism that reduces the frequency of encounters between non-nestmates.

21 However, due to confinement imposed by the nests' physical boundaries, it is likely that hosts

22 and inquilines would eventually come across each other. Under these circumstances, it is

23 plausible that inquilines would be required to behave accordingly to secure their housing. Here,

24 we tested this hypothesis predicting that, once inevitably exposed to hosts, inquiline individuals

25 would modulate their behaviour to circumvent conflict. While exploring the behavioural

26 dynamics of the encounter between both cohabitants, we find evidence for an unusual lack of

27 aggressiveness by inquilines towards hosts. Such a non-aggressive behaviour is characterised

28 by evasive manoeuvres that include reversing direction, bypassing and a defensive mechanism

29 using defecation to repel hosts. The behavioural adaptations we describe may play an intrinsic

30 role in the stability of cohabitations between termite species: by reducing the costs of conflicts

31 to both cohabitants, it may improve the chances for stable nest-sharing considerably.

32

33 Keywords: Cohabitation, Conflict, Inquilinism, Isoptera, Inquilinitermes microcerus,

34 Constrictotermes cyphergaster. 


\section{Introduction}

Nature provides innumerable opportunities to observe animals coexisting (Tokeshi, 2009; Gravel et al. 2011), from migratory species temporarily interacting with local communities (Kays et al., 2015) to organisms establishing long-term, interspecific relationships (Wilson, 1988). Of particular interest, the latter group includes species that cohabit a single place and may, therefore, interact several times throughout their lifespan. These associations, or symbioses (sensu De Bary, 1878; see Oulhen et al., 2016), often represent excellent opportunities to investigate how organisms with independent life-histories end up sharing precisely the same place. For instance, while making decisions about permanent housing, some organisms opt for nests already built by a different species, avoiding costs with construction. In addition to providing shelter, nests may contain resources continuously renewed over time (e.g. food and water) and attract a variety of opportunistic organisms on the way. That seems to be the case of termite nests (termitaria), in which is possible to find an impressive richness and abundance of non-nestmates cohabiting with the original termite builders (Costa et al., 2009; Monteiro et al., 2017).

Although a wide variety of species have been found inside termitaria (Kistner, 1969, 1979, 1990; De Visser et al., 2008), in this paper we focus on a remarkably distinct case of nest cohabitation between builder and invader, that is, a host termite and a secondary, opportunistic termite species so-called inquilines (sensu Araujo, 1970). It is worthwhile mentioning, however, that inquilinism among termites should not be mistaken with that occurring in Hymenoptera. Commonly referred as social parasites (Nash \& Boomsma, 2008), inquiline bees, wasps and ants tend to establish a close relationship with hosts and exploit their social behaviour intensively (Hölldobler \& Wilson, 1990). In termites, though, inquilines are thought to be primarily associated with the nest's physical structure itself, regardless of their association with host species (Shellman-Reeve, 1997; Marins et al., 2016). With proportionally smaller colonies and relatively low brood care (Korb et al., 2012) it is unlikely, although possible, that inquiline termites would deplete nest resources intensively, or exploit the host's social structure, as reported in different inquiline ant species (Buschinger, 2009).

Framing precisely inquilinism among termites into the spectrum of symbiotic interactions (e.g. parasitism, commensalism) can be challenging. For instance, although a number of studies have provided relevant information on different host-inquiline systems (e.g. Collins 1980; Redford, 1984; Eggleton \& Bignell 1997; Cunha et al. 2003; Costa et al. 2009;

Darlington, 2011; Cristaldo et al. 2012, 2014; Florencio et al. 2013; Campbell et al., 2016; DeSouza et al. 2016; Rodrigues et al. 2018), it remains unclear which costs (if any) inquiline termite colonies impose to host species. Even so, it seems plausible that a community of termite 
71 species within a single nest would be an ideal scenario for the emergence of conflict. Because

72 host termite species are known to respond aggressively towards a variety of nest intruders

73 (Emerson, 1938; Shellman-Reeve, 1997), the confrontation would arise predominantly from

74 encounters with non-nestmates. Aggressive behaviour seems to be, in fact, a default response of

75 the soldier caste of termites towards non-nestmates (Noirot, 1970), with individuals engaging

76 in endless fights while protecting their colonies (Binder 1987). Moreover, in addition to the

77 typical agonism of soldiers, hidden aggression among termite workers has been reported for

78 some species (Ishikawa \& Miura 2012).

79 Curiously enough, as opposed to hosts, inquiline colonies may be found in the wild

80 severely depleted in their contingent of soldiers (Cunha et al. 2003). The proportion of soldiers

81 in some cases may account for less than one per cent of the colony (HH, pers. obs.). Relying on

82 nest invasions to persist may, hence, represent a considerable risk for inquiline colonies, and an

83 intriguing question is how cohabitation in such terms is even possible. Not surprisingly,

84 previous works have tackled such an issue, suggesting proximate mechanisms that would allow inquilines and hosts to meet less frequently within the nest. In this regard, immediately after successful invasions, inquilines would establish themselves in the nest by decreasing chances of being noticed by hosts in the first place. Inquiline termites could achieve such an effect through various behaviours, including: (i) avoiding walking in galleries crowded by hosts (Grassé, 1986; Mathews, 1977); (ii) not conflicting with dietary requirements of the host (Miura \& Matsumoto, 1997; Florencio et al., 2013); (iii) intercepting hosts' chemical signals and using the information acquired to preclude encounters with hosts (Cristaldo et al., 2014, 2016a); and (iv) keeping the colony isolated from hosts by changing the nest structure (e.g. building their own galleries and sealing chambers: $\mathrm{HH}$, pers. obs.). Although functioning through independent mechanisms, these behavioural strategies seem to coincide in a single outcome: by preventing direct contact, inquilines reduce the frequency of encounter with hosts.

As efficient as it may seem, however, while such strategies could potentially attenuate conflictual events, they would not entirely prevent encounters from happening. For most inquiline species (including the one studied here), there is no evidence yet of colonies exiting nests after they break in, neither for nest defence nor for foraging. The only known exception is

100 the winged reproductive caste that leaves the nest during swarming (Matsuura, 2010). These

101 facts together suggest that there is an associated probability of interspecific encounter to be considered. Besides, the confinement imposed by the nest's physical boundaries would keep

103 individuals locally restricted and bound to meet in the long-term. Under these circumstances

104 inquilines would be required to behave accordingly, for instance, mitigating detrimental

105 consequences of aggressive encounters with nest owners. 
Although hinted in the past, this intuitive, theoretical prediction was never directly

107 tested, and little is known about host-inquiline dynamics within the nest, or to what extent

108 inquiline strategies are sufficient to cope with the menace of imminent confrontation with hosts.

109 This information could provide important clues about how these cohabitations hold in nature. A

110 conservative approach to this issue would sustain that inquilines should replicate, at the

111 individual level, the evasive behaviour they exhibit as a group. In this context, one would expect

112 the strategies to avoid conflict (highlighted above) to be mere consequences of a non-

113 threatening posture exhibited by inquiline individuals. Here, we tested this hypothesis

114 predicting that, once inevitably exposed to hosts, inquiline individuals would adopt a non-

115 aggressive posture and modulate their behaviour to a less threatening profile. As a result, the

116 colony would be able to reduce conflict with nest owners collectively. Such an assumption

117 would imply that inquiline individuals should weaken conflict escalation by (i) being lethargic

118 and minimising encounters with hosts and (ii) exhibiting low aggressiveness by avoiding either

119 initiating or retaliating attacks.

120 To test these assumptions, we observed in detail the behaviour of an obligate inquiline

121 termite, Inquilinitermes microcerus Silvestri (1901) (Termitidae: Termitinae), in the presence of

122 its host termite, Constrictotermes cyphergaster Silvestri 1901 (Termitidae: Nasutitermitidae).

123 We exposed species to each other under two different experimental scenarios: (i) in closed

124 arenas, as to keep individuals locally restricted and favour host-inquiline encounters; and (ii) in

125 open arenas, where inquilines had a chance to flee from hosts. By compiling full ethograms for

126 the encounter between I. microcerus and C. cyphergaster, we add new information to the current

127 knowledge on nest-sharing termite species. These descriptions highlight relevant aspects to

128 consider while studying the underlying mechanisms of coexistence between species living in

129 environments circumscribed by discrete physical barriers. Furthermore, we argue that the

130 behavioural profiles here described lend support to the notion of inquilines as peaceful guests,

131 contributing to the growing view of conflict-avoidance as an effective strategy to coexist in

132 harsh environments. 


\section{Methods}

\section{Biological model}

136 The termite $C$. cyphergaster (hereafter, host) is a Neotropical species widely distributed 137 in South America (Mathews, 1977; Krishna et al., 2013) known to forage at night in exposed

138 columns and without the protection of covered galleries (Moura et al., 2006). In this species,

139 nest foundation starts on the ground with a royal couple, and after reaching a certain size,

140 colonies migrate to the trees, where they establish typical arboreal nests (Vasconcellos et al.,

141 2007). At this phase, it is usual to find colonies of I. microcerus, a secondary opportunistic

142 termite species that inhabit the nests (hereafter, inquilines). Such a suggestive name as

143 Inquilinitermes has its reasons: inquilines seem to be unable to build nests by their own

144 (Emerson, 1938; Mathews, 1977), being found so far exclusively within host nests. Although it

145 remains unclear how exactly nest invasion occurs, there seems to be a critical nest volume

146 above which inquiline colonies are more likely to be found within host nests (13.6 L, see

147 Cristaldo et al., 2012). The nest's size seems to indirectly affect inquilinism in termites, being

148 negatively related to defence rates (DeSouza et al., 2016). Besides, while evaluating populational

149 parameters of nests containing inquiline colonies, Rodrigues et al. (2018) reported a negative

150 correlation between the number of individuals and the proportion of soldier/workers.

151 Compared to hosts, inquiline colonies are much smaller in size, but still easily detectable due to

152 a characteristic dark lining covering their galleries (Cunha et al., 2003; Cristaldo et al., 2012;

153 Florencio et al., 2013). Inside the nest, inquiline colonies are often associated with chambers

154 filled with a black material, hypothesised in the past as waste dumped by hosts (Emerson,

155 1938), but still of unknown origin.

\section{Study site and collection}

158 To carry out experiments, 27 host nests with inquiline colonies were collected from two

159 locations in the Brazilian Cerrado (Ratter, Ribeiro, \& Bridgewater, 1997): 15 nests collected

160 near the municipality of Sete Lagoas (19²7'57"S, 4414'48"W) in July 2012; and 12 nests

161 collected near the municipality of Divinópolis (200ㅗ'20"S, 44ํ5' $\left.02^{\prime \prime} \mathrm{W}\right)$ in January 2015. Both

162 sites, located in Southeastern Brazil (State of Minas Gerais), have climate resembling savannas

163 (Aw, in Köppen-Geiger classification), and are subjected to an equatorial climate with dry

164 winters (Aw) (Kottek et al., 2006). 
166

167

168

169

170

171

172

173

\section{Experimental design}

In order to access behavioural profiles at host-inquiline encounters, cohabitants were

taken from their nests, acclimatised for 30 minutes in separate containers, and then gathered in arenas for video recording (Fig. 1). Experimental arenas consisted of plastic Petri dishes (Ø $53 \mathrm{~mm}$ ) lined with paper (Whatman $\mathrm{N}^{\circ} 1$ ), and video-samples were taken with a digital camera (Nikon D300S, 720p, 25fps). All videos were recorded under visible light, and the room temperature was controlled between $23^{\circ} \mathrm{C}$ and $25^{\circ} \mathrm{C}$.

\section{(A) CLOSED ARENAS}
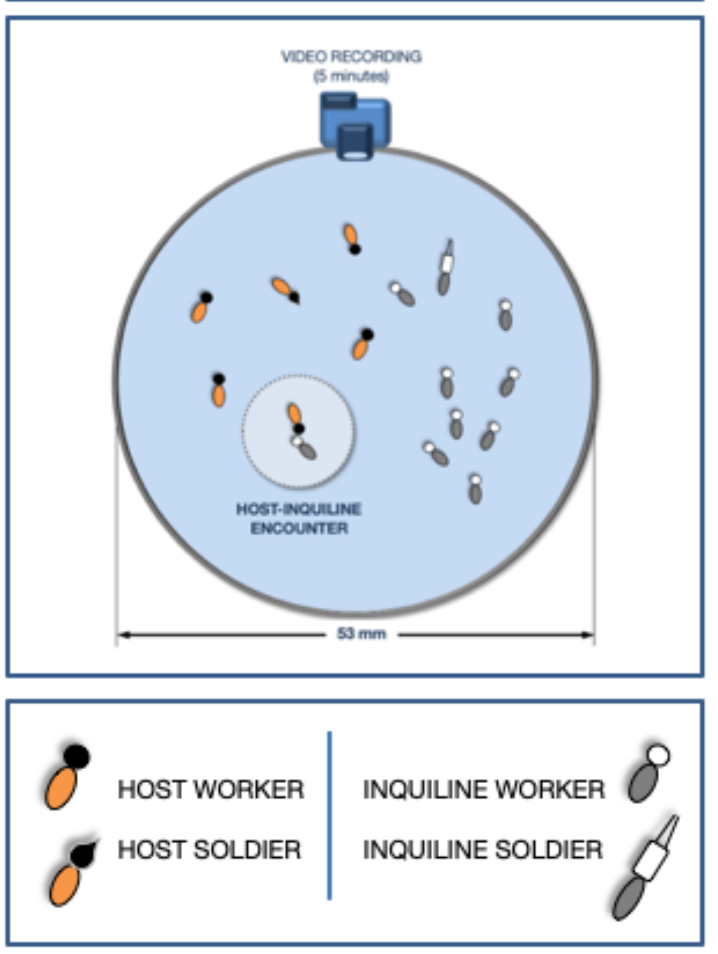

(B) OPEN ARENAS

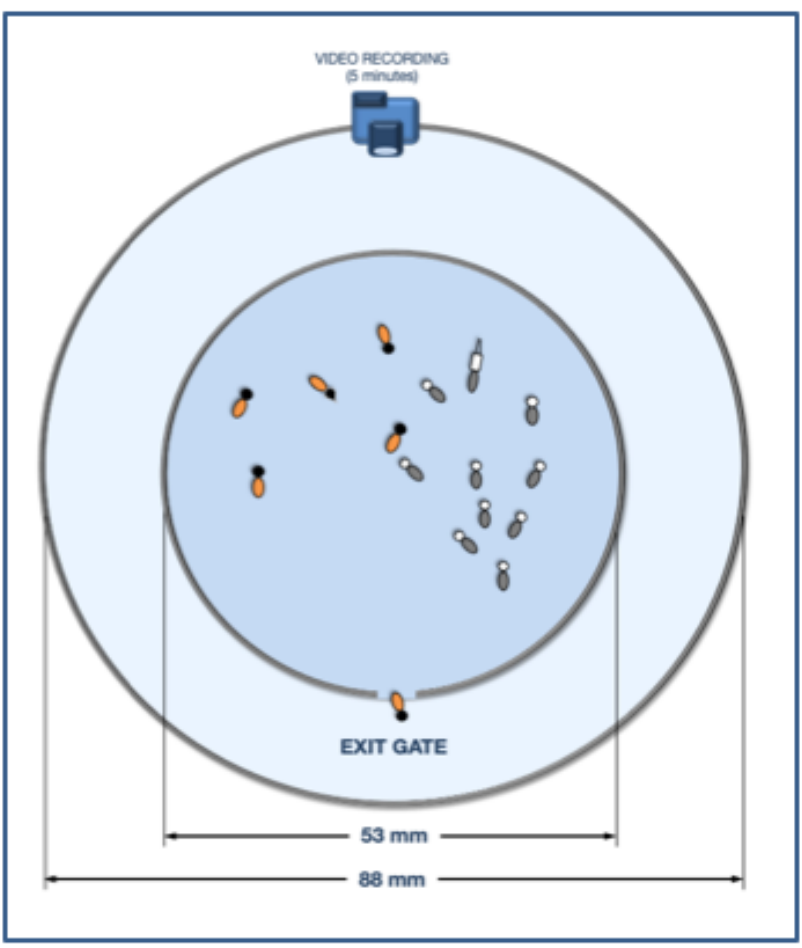

Figure 1. Arena settings for video recording: (A) Closed arenas, (B) Open arenas. We recorded videosamples of five minutes (300 seconds) for focal animal observation, which was carried out by observers using a 14" LED-LCD 1080p screen; an example of a heterospecific encounter is highlighted by a lighter circle within the closed arena (A). The internal area had a diameter of $53 \mathrm{~mm}$. The external area had a diameter of $88 \mathrm{~mm}$. A gate consisted of a single opening with diameter of $3.5 \mathrm{~mm}$ on the arena wall connecting internal and external areas.

We designed two experiments to test our predictions. In the first experiment (Fig. 1A), host and inquiline individuals were mutually confined in closed arenas, a locally restricted condition intentionally designed to improve the chances of an encounter between the species. In the second experiment, hosts and inquilines were gathered in arenas mostly identical to those used in the first setup, except for the presence of an exit gate. This gate consisted basically of a single opening ( $\varnothing 3.5 \mathrm{~mm})$ on the arena wall, giving access to an external circular area ( $\varnothing 88 \mathrm{~mm})$ 
188 encompassing the inner one ( $\varnothing 53 \mathrm{~mm}$ ). This second experiment was conducted to inspect

189 whether inquilines would (i) remain idle or (ii) move away when given a chance to flee from

190 host aggressions (Fig. 1B). The latter response could potentially lead to spatial segregation

191 between species, a result that would be in line in field observations.

192 We defined two treatments using open arenas to test whether the presence of inquilines

193 would affect how hosts explore the space available: (i) open arenas containing host and

194 inquilines; and (ii) open arenas containing only hosts, as a control. Experiments were conducted

195 with individuals kept under optimal density (0.12; for details, see Miramontes \& DeSouza, 2008)

196 and in a worker-to-soldier ratio similar to that found in natural conditions (Cunha et al., 2003).

197 Experimental groups, therefore, contained: (i) one soldier and four workers for hosts and (ii)

198 one soldier and nine workers, for inquilines. Individuals composing a given experimental group

199 were never present in a second trial, as to avoid interference from prior contact with non-

200 nestmates.

\section{Behaviour annotation and observational protocol}

To determine observable behaviours of relevance to our scope, we performed the

204 following procedure: before the main experiments, preliminary observations were taken as to

205 detect behaviours possibly performed by individuals. Video-samples used to perform these

206 observations were never reused in the main experiments. At this phase, we spent efforts to

207 describe as many behaviours as possible. Because extensive behavioural descriptions may

208 contribute to misleading observation (Lehner, 1998), we created a flowchart with simple,

209 straightforward labels hierarchically organised (Fig. 2). Observers used this diagram and a list

210 of short behavioural descriptions (Table 1) as a reference for annotation.

212 Table 1. Behavioural description based on preliminary observations. We defined nine observable

213 behaviours of relevance to our scope using ten additional video-samples. For statistical analysis, we

214 classified each behaviour in two ways: (i) either as within-species or between-species; and (ii) either as

215 aggressive or non-aggressive.

216

\begin{tabular}{|c|c|c|c|}
\hline Behaviour & Description & Type of encounter & Type of interaction \\
\hline RESTING & Focal animal remains stationary at the same place & non-aggressive & within-species \\
\hline WALKING & Focal animal moves freely around the arena & non-aggressive & within-species \\
\hline ANTENNATING WALL & Focal animal reaches the arena wall and performs only antennation & non-aggressive & within-species \\
\hline ANTENNATING NESTMATE & Focal animal encounters nestmate and performs only antennation & non-aggressive & within-species \\
\hline ANTENNATING NON-NESTMATE & Focal animal encounters non-nestmate and perform only antennation & non-aggressive & between-species \\
\hline IGNORING & Focal animal encounters non-nestmate and do not react & non-aggressive & between-species \\
\hline REVERSING & Focal animal encounters non-nestmate and perform u-turn manoeuvre & non-aggressive & between-species \\
\hline PASSING & Focal animal encounters non-nestmate and perform a bypass manoeuvre & non-aggressive & between-species \\
\hline ATTACKING & Focal animal encounters non-nestmate and performs aggression & aggressive & between-species \\
\hline
\end{tabular}




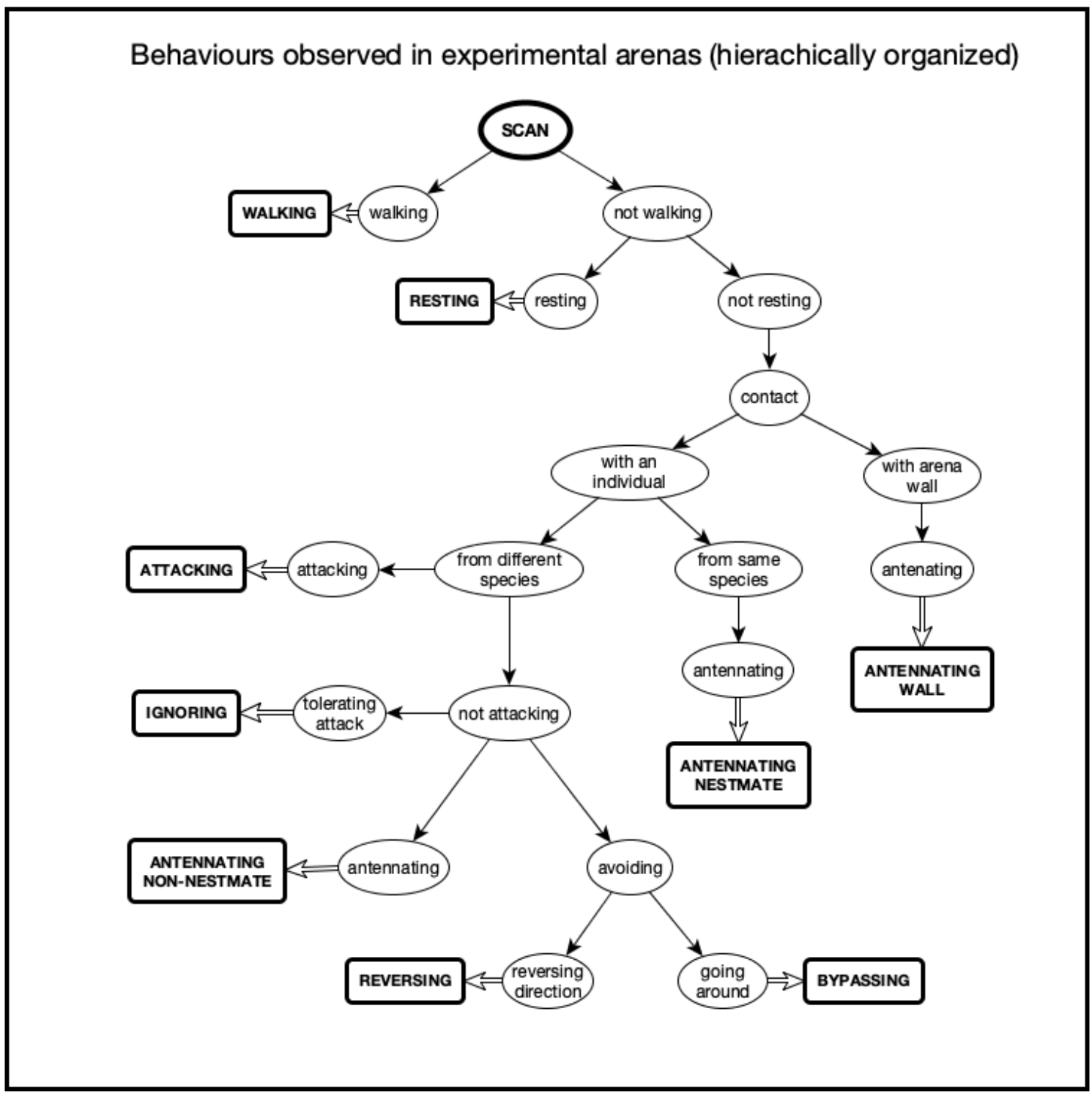

219 Figure 2. Behavioural flowchart used for annotation. Labels are hierarchically organised to allow

220 stepwise classification of behaviours. We defined nine observable behaviours in preliminary

221 observations. Combined to a behavioural description presented in Table 1, this flowchart served as a

222 reference for annotation.

We adopted focal animal sampling (Altmann, 1974) with observations taken from videosamples to capture interactions between hosts and inquiline individuals. We recorded a total of

22620 video-samples of five minutes (10 using closed arenas, 10 using open arenas). Behaviours

227 performed by host workers (HW), host soldiers (HS), inquiline workers (IW) and inquiline

228 soldiers (IS) were annotated for each video-sample using the flowchart described above. For

229 each one of these categories, an individual was arbitrarily selected for focal observation

230 (hereafter, focal animal). Using a 14" LED 1080p screen to watch video samples, we took three-

231 seconds observations (hereafter, scans) for each focal animal. Scans were taken at regular time

232 intervals of 10 seconds, indicated to observers by scheduled sound signals. This method 
233 provided 31 scans per focal animal for each video-sample. Finally, we organised all behavioural

234 annotation in files including all relevant information (e.g. observer, date and time of recording,

235 room temperature)data analyses.

\section{Measuring aggressiveness and host-inquiline interactivity}

238 To measure host's and inquiline's aggressiveness in closed arenas, we classified

239 behavioural observations into two mutually exclusive categories regarding the type of

240 encounter: (i) aggressive encounter, when focal animals encountered non-nestmates and

241 performed aggression (i.e. attack); and (ii) non-aggressive encounter, when focal animals

242 interacted with non-nestmates but did not perform aggression (i.e. antennating non-nestmate,

243 reversing, bypassing, ignoring). To measure interactivity between hosts and inquilines in closed

244 arenas, we classified behavioural observations into two mutually exclusive categories regarding

245 the type of interaction: (i) within-species, when focal animals performed actions either by

246 themselves (i.e. resting, walking, antennating wall) or with nestmates (i.e. antennating

247 nestmate); and (ii) between-species, when focal animals performed actions after establishing

248 physical contact with non-nestmates (i.e. antennating non-nestmate, ignoring, bypassing,

249 reversing, attacking).

\section{Assessing behavioural profiles}

252 To analyse the influence of specific behaviours in the general profile of hosts and

253 inquilines, we developed a network analysis using the free software yEd Graph Editor version

2543.14 .4 (yWorks, 2015). To build graphs for each caste, we performed the following procedure:

255 using behavioural sequences extracted from annotations, we constructed adjacent matrices

256 containing the behavioural change for each caste (Supplementary Material, Table S2), which

257 later was imported to yEd to draw the graphs. As typically done in standard network analysis

258 (Newman, 2003), graphs consisted of networks of nodes linked by connecting edges (i.e.

259 directional arrows). In our case, however, nodes represented specific observable behaviours

260 executed by individuals, whereas connecting edges represented behavioural changes from a

261 given behaviour to another one. That is, if individuals changed from rest to walking behaviour,

262 the behavioural change annotated would be rest-walk. With nine observable behaviours defined

263 in our scope (Table 1), 81 types of behavioural change could be possibly observed. With the

264 constructed graphs, we calculated centrality measures (Freeman, 1978) using the number of

265 incoming connecting edges for each node (Brandes \& Erlebach, 2005). Then, using the

266 calculated centrality scores, we adjusted the size of nodes to visually represent the degree of

267 influence exerted by each behaviour upon profiles (i.e. the larger the size of a node, the higher

268 its influence on the network). 


\section{Ethogram validation}

We adopted a procedure suggested by Dias et al. (2009) to validate our ethograms.

271 Following this approach, we used behavioural accumulation curves (BAC) to assess an optimal

272 balance between (i) effort with sampling and (ii) ethogram completeness. A minimum of 250

273 independent observations would be required to efficiently capture a total of ten observable

274 behaviours (Fig. 3). In our study, we extrapolated this number and performed 1240 discrete

275 observations for the nine observable behaviours previously defined (that is, 31 scans $\mathrm{x} 2$ castes

$276 \times 2$ species $\times 10$ replicates $=1240$ scans $)$.

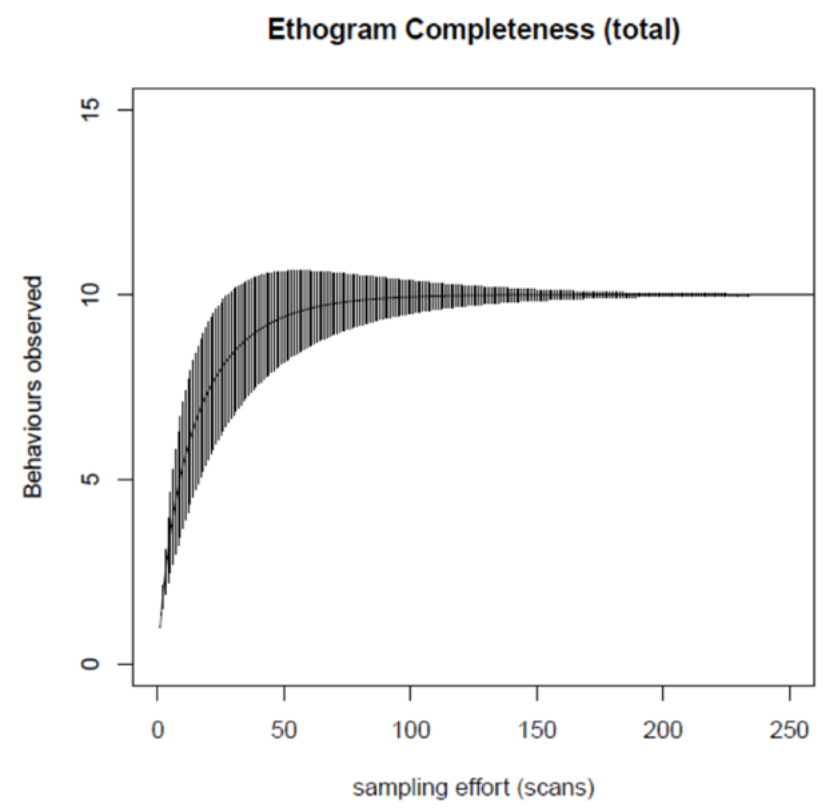

278 Figure 3. Ethogram completeness using Behavioural Accumulation Curves (Dias et al., 2009): the X-axis 279 represents sampling effort, that is, the number of scans performed to observe all behaviours (in our case, 28031 scans $\times 2$ castes $\times 2$ species $\times 10$ replicates $=1240$ scans); the $Y$-axis represents the accumulative number of behaviours experimentally observed in trials.

\section{Statistical analyses}

We performed the statistical analyses in R, version 3.5.2 (R Development Core Team, 2018) using Generalized Linear Modelling (GLM) under Binomial errors with log-link. As a conservative approach, the significance of treatments was accessed using the following procedure: we compared complex models to simpler ones achieved by combining treatment

288 levels (Crawley, 2012). When simplification did not provoke significant changes, simpler models

289 were accepted, and the combined treatments were considered equivalent to each other. We

290 then submitted adjusted models to a residual analysis as to check the suitability of the

291 modelling equation and normality of error distribution. If required, error distribution was

292 adjusted using Quasi-binomial distribution. In all tests conducted, we considered an $\alpha=0.05$ to

293 assess statistical significance. 


\section{Results}

\section{Inquilines suffered attacks from hosts but responded with low aggressiveness}

In closed arenas, the proportion of aggressive interactions initiated by hosts when encountering inquilines was significantly higher than the proportion of non-aggressive interactions ( $G L M ; \mathrm{F}_{1,98}=16.72, P<0.001$; Fig. 4). As expected, caste was determinant in the type of aggression inflicted by individuals. While host workers physically injured inquilines by biting them in several portions of their softy bodies, host soldiers frequently adopted an agonistic display, characterised by an abrupt movement with stretched antennae. In C. cyphergaster, soldiers present a snout-like protuberance in their head that contains a frontal gland. Such an apparatus produces a mixture of terpenoids, often used against targets in defensive actions (for details, see Cristaldo et al., 2015). In this study, however, we were not always able to detect whether an agonistic display was followed by chemical spray.

When attacked by hosts, the proportion of aggressive reactions initiated by inquilines was not significantly higher than the proportion of non-aggressive interactions (GLM; $F_{1,98}=1.74$, $\mathrm{P}=0.18$; Fig. 4). When threatened, or even severely injured by hosts, inquiline workers never retaliated (attacking; Fig. 5). Instead, individuals were more likely to adopt evasive manoeuvres and quickly divert from aggressors. These actions occurred immediately after an active contact with host individuals was established, and included behaviours that avoided the opponents

313 (reversing, bypassing; Fig. 5). Besides escaping from host threats, inquiline workers also

314 performed ignoring behaviour. In this case, individuals actively touched by hosts did not react 315 to such a stimulus, remaining completely stationary (ignoring; Fig. 5).

Hosts

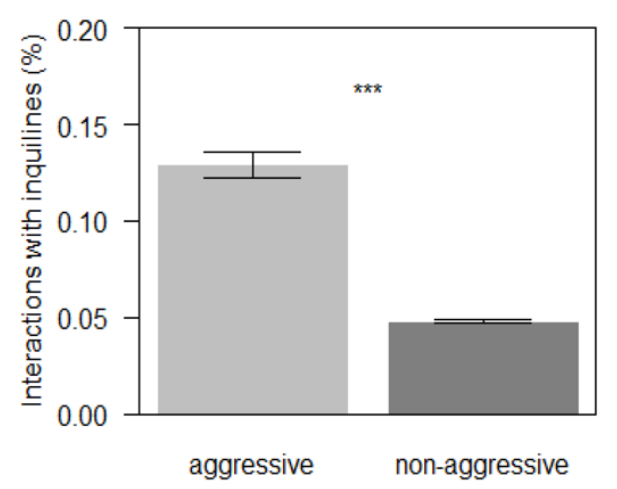

Inquilines

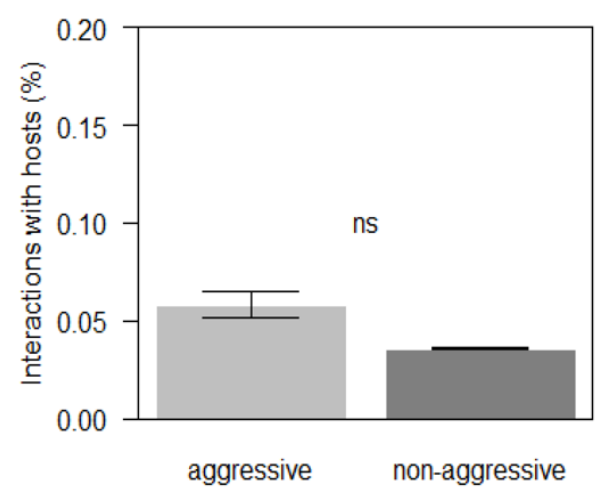

317 Figure 4. Interactions observed in closed arenas for hosts, C. cyphergaster (right), and inquilines, I.

318 microcerus (left). Proportions were calculated by the number of aggressive and non-aggressive 319 interactions observed, divided by the total number of observations taken from video-samples $(\mathrm{N}=10)$.

320 Behaviours that do not preclude interaction (i.e. resting, walking and antennating wall) are not 321 represented. For this reason, frequencies do not sum up to $100 \%$. Light bars: aggressive interactions;

322 Dark bars: non-aggressive interactions. 

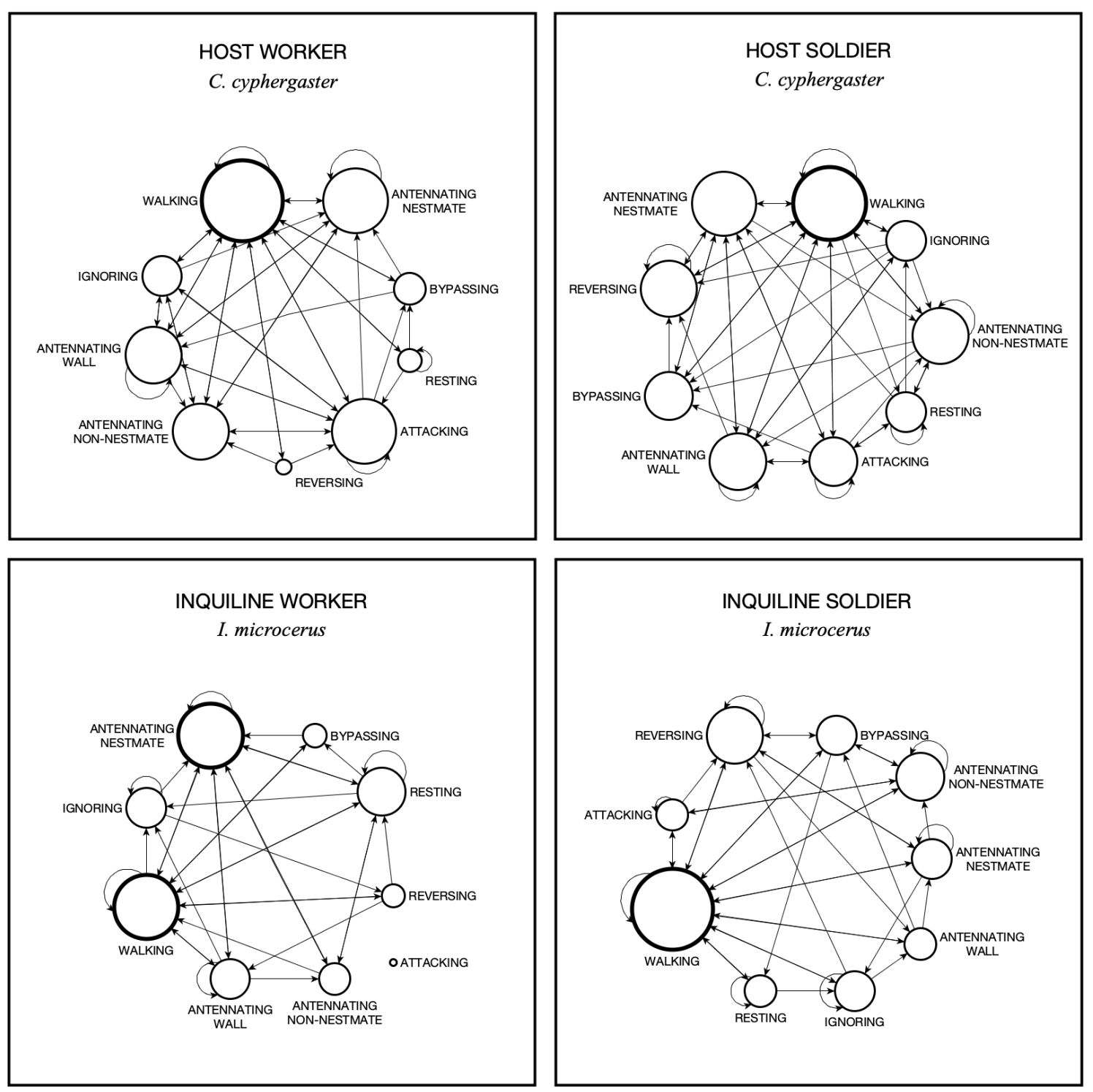

324 Figure 5. Behavioural profiles observed for each caste. Nodes represent behaviours performed by individuals, whereas connecting edges (arrows) represent behavioural changes occurred from one behaviour to another. Behaviours with the highest influence on the network are highlighted with thicker node contours. Node size was adjusted using calculated centrality measures to visually represent the degree of influence exerted by each behaviour upon the profiles. Obs.: For inquiline worker, attacking never happened and, therefore, this behaviour is not connected to the network. A version containing calculated scores is included in the Supplementary material (Figure S1). among inquilines. As opposed to inquiline workers, inquiline soldiers performed aggression in retaliation to host assaults (Supplementary Material, Video S1), even though this occurred not so frequently (attacking, Fig. 5). Aggression performed by inquiline soldiers consisted of snapping attacks, a sudden release of slender mandibles pressed against each other producing powerful strikes over opponents (Supplementary Material, Video S2). 


\section{Inquilines interacted little with hosts even when locally restricted}

Inquilines exhibited low interactivity with host individuals, even when locally restricted and presumably more prone to meet, such as in closed arenas. The proportion of betweenspecies observations was considerably lower than the proportion of within-species observations $\left(G L M ; \mathrm{F}_{1,178}=71.73, P<0.001\right)$. Besides, regarding the behavioural change, inquiline workers exhibited a loop between resting, walking and antennating nestmate, all behaviours with no contact with the host (Figure S3). The Table S1 of Supplementary Material contains absolute numbers for between- and within-species observations for each one of the castes.

\section{Hosts were active in arenas, while inquilines were lethargic}

When placed in closed arenas, host individuals performed antennation on the arena wall more frequently than inquiline individuals (GLM; $F_{1,78}=4.73, P<0.005$ ), an indication that hosts could be possibly attempting to broaden their patrolled area. We confirmed this suspicion with results from the second experiment, with open arenas: host individuals quickly moved to the external area passing through the gate as soon as they found it. Either in the presence or absence of inquiline individuals, there was no difference in the mean time spent by host individuals to leave the internal area $\left(19.93 \pm 3.56\right.$ seconds, $\left.F_{1,9}=0.34, P=0.57\right)$, meaning that inquilines did not threaten hosts. Inquilines, in turn, were more prone to remain stationary and never left the internal area, a result that seem to confirm the putative lethargic behaviour of inquilines.

\section{Inquiline's defecation prevented host aggression}

We observed an unexpected response among inquiline workers: when threatened by hosts, inquiline workers deposited faecal pellets always towards the direction from which they suffered threats (Supplementary Material, Video S3). Rather than usual defecation, this behaviour seemed to be elicited by host aggressions in a particular way: when receiving attacks from backwards, individuals immediately placed faecal pellets in front of aggressors and escaped forward. Threats coming from any other direction, however, triggered a slightly different response: before defecating, individuals first adjusted their posture accordingly, placing themselves in a way that they could quickly drop faecal pellets in front of the aggressors. Only after such a move, inquiline workers defecated and escaped forward. We observed this behaviour 33 times, and in all occurrences, faecal pellets immediately prevented inquilines of being chased of receiving further attacks from aggressors. Although we did not measure whether inquiline faeces have a repellent effect over hosts, it was evident in our recordings that 
373 areas containing faeces were less visited by host individuals (Supplementary Material, Video

374 S3).

\section{Caste types showed unique behavioural profiles}

We found striking differences when comparing the behavioural profile of hosts and inquilines (Fig. 5). Visual representations obtained from network analyses revealed unique configurations for each of the castes analysed. For all caste types, walking was the behaviour with the highest centrality score (walking; Fig. 5), that is, with the highest influence. The only caste type that presented two behaviours equally influential to the network was that of inquiline workers. In this group, besides walking, conspecific antennation also reached the highest centrality score (antennating nestmate; Fig. 5). As highlighted above, because inquiline workers never performed an attack, this behaviour presented the lowest centrally score and did not connect to the network (attacking; Fig. 5).

\section{Discussion}

In environments where individuals are constantly surrounded by potential aggressors, the evolution of a "peaceful behaviour" may appear, at first, counter-intuitive. Our results demonstrate, however, that for inquilines (I. microcerus) non-aggressive behaviour is a valid strategy that mitigates detrimental consequences of unexpected encounters with hosts $(C$. cyphergaster). More important, it seems to secure housing for inquiline colonies within host nests in the long-term. Evasive behaviour by inquilines has been previously suggested as one of the proximate causes in inquilinism, as it reduces the frequency of encounter between colonies. Here, besides providing substantial behavioural data supporting this idea, we showed that once inevitably exposed to hosts, inquiline individuals can modulate their behaviour to a less threatening profile and circumvent confrontation. As compared to other termite species, this suggests a degree of adaptation towards a more flexible behaviour which could, in turn, strongly

\section{The behavioural adaptations of a peaceful guest}

A set of behaviours seem to support our interpretation of inquilines as peaceful guests within host nests. First, when encountering host individuals, inquilines suffered several attacks but did not react with the same level of aggressiveness. Lack of aggression was markedly evident among inquiline workers. This caste not only never performed a single attack during our experiments but also managed to move away from aggressors with evasive manoeuvres

408 (reversing, bypassing; Fig. 5). The same was not observed for inquiline soldiers, who did 
409 retaliate host attacks with snapping (attacking; Fig. 5). It is worth mentioning, however, that

410 soldiers are rare in natural colonies of I. microcerus (Cunha et al., 2003), and sometimes even

411 absent (HH, pers. obs.). Thus, it is unlikely that the aggressive behaviour of a minority would

412 contribute to increase the species aggressiveness substantially. Although we did not find

413 evidence pointing in such a direction, we acknowledge the necessity of more studies evaluating

414 the interplay between worker's and soldier's behaviour in field conditions, something that was

415 clearly beyond the scope of the present work.

416 A second behaviour linked to the levels of aggressiveness reported (Fig. 3) was the

417 reduced mobility of inquilines. Consequently, the interaction between hosts and inquilines in

418 arenas was limited. In open arenas, only hosts moved to the external area over time, whereas

419 inquilines remained quiet in the inner portion. Such spatiotemporal segregation could be a

420 direct consequence of the behavioural profile of hosts. As we have shown, walking was a

421 commonly performed behaviour among all castes, but at the same hosts spent less time

422 remaining stationary in the same place (resting, Fig. 5). Theoretically, as they walk more

423 intensively and explore sites more efficiently, gates would be more readily found. In this regard,

424 the presence or absence of non-nestmates in arenas did not affect the time spent by hosts to

425 pass through the gate and access the external area. This result indicates that inquilines did not

426 necessarily triggered the collective motion of hosts to the external area. Dynamics of both

427 collective behaviour and environment have been suggested to regulate group-level properties in

428 ants (Gordon, 2019). In termites, some studies have explored principles of collective behaviour

429 (Sumpter, 2006) using agent-based models to understand self-organisation of groups, from nest

430 construction processes (Deneubourg, 1977) to aspects of social facilitation (Miramontes \&

431 DeSouza, 1996; DeSouza et al., 2001). Still, for nest-sharing termite species, to what extent

432 individual behaviour shape collective motion patterns, remains a topic to be fully understood.

433 A third component that seemingly affected the amount of aggression we observed in

434 arenas was defecation by inquilines. Presence of faecal pellets shortened host-inquiline contact

435 in virtually all occasions, and consequently, host attacks towards inquilines were less frequent.

436 This result indicates that faeces may improve evasion by preventing host aggressions. In fact,

437 defecation as an evasive mechanism is not exclusive of $I$. microcerus, being first described for

438 termites by Coaton (1971) in Skatitermes sp. (Termitidae: Apicotermitinae). Such a defensive

439 behaviour may have important implications for cohabitation: if faeces indeed repel hosts, single

440 pellets placed in narrowed galleries throughout the nest could prevent host contact in a very

441 efficient, inexpensive way. Besides, it is possible that while placing the pellets, I. microcerus

442 would be spreading their scent throughout the entire nest, making it harder for hosts to locate

443 the core of their colonies. Accordingly, while studying the cohabitation of another host-inquiline

444 pair (C. cavifrons and I. inquilinus, respectively), Jirošová et al. (2016) showed that walls from 
445 the inquiline portion of nests contain levels of C12 alcohols, a repellent for host individuals.

446 According to these authors, chemically mediated spatial separation of hosts and inquilines may

447 aid to avoid conflict. Among other non-related groups, such as the cuckoo bumblebee, repellent

448 odours are known to reduce host attacks (Lhomme et al., 2012), suggesting that this is an

449 effective mechanism across taxa.

\section{The meaning of an interspecific encounter}

The non-aggressive behaviour observed among inquilines raises the question of

whether such a strategy would be useful within the nest. After all: are encounters with the host species a real threat for inquiline colonies? We provide evidence that there are, indeed, mandibles, are the ones who inflict the physical damage. In C. cyphergaster, terpenoids sprayed from the frontal gland of soldiers function as an effective alarm pheromone (Cristaldo et al. 2015). Thus, once a target is sprayed, it recruits nestmates to converge upon the site and deploy themselves around it (Eisner et al., 1976). In such a harsh environment, where virtually all individuals are potential aggressors, it is plausible that a peaceful behaviour, rather than a costly aggressive profile, could be a simpler alternative solution. All in all, as compared to a belligerent set of behaviours, a non-threatening profile would demand less elaborated actions, plausibly resulting in lower activity and reduced probability of interspecific encounter.

\section{The mechanism of conflict avoidance}

Behaviours preventing confront escalation are widespread. When attacked by host ant workers, for instance, parasite ant queens do not react aggressively and, instead, quickly move towards the fungus garden remaining quietly there (Nehring et al., 2015). In another typical social parasite, Maculinea rabeli (Lycaenidae), larvae individuals are known to suppress aggression from their host ants by mimicking aspects of the brood's pheromone (Akino et al.,

474 1999; Pierce et al., 2002). Among bees, changes in the host's behaviour towards non-aggressive 475 types have been also reported: in the presence of the cuckoo bumblebee Bombus vestalis, host colonies decrease worker aggressiveness towards alien individuals, possibly due to changes in the host worker's discrimination (Lhomme et al., 2012). Examples of aggressiveness being

478 affected by external factors are not exclusive to social insects, extending to other invertebrate 479 and vertebrate groups (Aureli et al., 2002; Baan et al., 2014; Gobush \& Wasser, 2009; Thierry et 480 al., 2008). In termites, aggressiveness may depend on factors such as diet (Florane et al., 2004), 
481 caste ratios (Roisin et al., 1990), nestmate recognition (Delphia et al., 2003; Haverty \& Thorne, 482 1989), group composition (Haverty \& Thorne, 1989), territoriality (Adams \& Levings, 1987;

483 Levings \& Adams, 1984) and resource availability (Cristaldo et al., 2016b). Even inter-colony

484 aggression, presumably more predictable due to higher relatedness, is not always consistent

485 (Binder, 1987). Species may exhibit behavioural plasticity (Ishikawa \& Miura 2012), responding

486 aggressively in some cases (Su \& Haverty, 1991), and lacking aggression in others (Delaplane,

487 1991; Neoh et al. 2012). Altogether, these reports indicate that it is possible to have scenarios in

488 which termite species adopt low aggressiveness profile, rather than the typical aggressiveness

489 observed among the group.

490 The symbiosis between C. cyphergaster and I. microcerus is a case of obligate

491 inquilinism, meaning that at least for inquilines, nest-sharing has become mandatory (Shellman-

492 Reeve, 1997). Evolutionary costs and drawbacks of such a specialisation by inquilines remain to

493 be assessed, although the benefits associated with nest invasion seem to be straightforward:

494 nest invaders are not required to spend time and energy building their own home. At the same

495 time, being nest construction a demanding, costly process (Korb \& Linsenmair, 1999), one

496 would expect such inquiline invasions to be not strictly in the interest of hosts. In this sense, it

497 would be reasonable to think of a scenario in which hosts would endeavour to detect inquilines,

498 whereas inquilines would try to go unnoticed by hosts. Under such driving forces, it is possible

499 that an evolutionary arms race would take place (Dawkins \& Krebs, 1979), leading hosts and

500 inquilines to reach well-adjusted behavioural profiles. In doing so, both cohabitant would

501 become highly specialised in their neighbour (Kilner \& Langmore, 2011).

\section{Cohabitation and conflict}

504 Cohabitation goes way beyond the "living in overlapping spaces". Instead, it is a result of

505 multiple interactions over time. Whether interactions contribute for the emergence of stable

506 relationships depends on the consequences mutually inflicted by the parties involved. With our

507 approach, we presented findings supporting a notion that hostile interactions do not always

508 lead to increased aggressiveness between opponents, especially if asymmetric aggression or

509 lack of reciprocal retaliation is in place. Although a common event in nature, conflict can be a

510 limiting factor for species coexisting. While surpassing acceptable thresholds, excessively high

511 levels of aggression can jeopardise relationships between organisms and lead entire colonies to

512 collapse. The behavioural adaptations we described, seem to allow inquilines to manage the

513 amount of aggression received from their hosts. Such a non-threatening individual behaviour

514 may play a fundamental role in cohabitation, as it seems to increase the chances of a stable

515 (although asymmetric) relationship between host and inquiline colonies considerably. We

516 suggest that further research should explore the contributions of such individual actions on 
517 collective patterns in the system. While in line with previous reports on cohabitation between

518 termite societies, our findings reinforce the growing view of conflict management as a critical

519 component of socially complex systems. Finally, descriptions of peaceful mechanisms by

520 recipients of aggression in locally restricted, hostile environments should contribute to putting

521 conflict and its consequences in a broader perspective, adding novel insights for studies

522 involving multiple group-living organisms.

\section{Acknowledgements}

525 We thank Dr Diogro A. Costa, Dr Alessandra Marins and Dr Vinícius B. Rodrigues for useful

526 discussions; Júlio H. Santos and Kátia C. D. Santos for helping with data collection; Dr Fernando

527 Valicente from the Brazilian Enterprise for Agricultural Research (EMBRAPA) and Prof. Ângelo

528 Fonseca for all logistic support. This study was partially funded by (i) the National Council of

529 Technological and Scientific Development (CNPq), (ii) the Foundation for Research of the State

530 of Minas Gerais (FAPEMIG: APQ-08811-15 and BPV-00055-11) and (iii) the DFG Centre of

531 Excellence 2117 "Centre for the Advanced Study of Collective Behaviour" (ID: 422037984). HH

532 was supported with a scholarship from the Foundation for Research of the State of Minas Gerais

533 (FAPEMIG) and a Research grant from the Deutscher Akademischer Austauschdienst (DAAD).

534 PFC holds a Research Fellowship from Coordination for the Improvement of High Education

535 Personnel (CAPES; PNPD no.1680248). ODS holds a Research Fellowship from CNPq (PQ

$536305736 / 2013-2)$. This paper is a contribution from the Lab of Termitology, Federal University

537 of Viçosa, Brazil (http://www.isoptera.ufv.br), deriving from HH’s MSc thesis.

\section{Author contribuitions}

540 HH, PFC \& ODS conceived the study; HH \& PFC developed fieldwork, behavioural recording and

541 ethogram design. HH conducted data collection, statistical analysis and drafted the paper. PFC \&

542 ODS commented on the manuscript, improving it considerably for submission.

\section{Ethical statement}

545 We obtained all required permits for the present study, thus complying with relevant

546 regulations governing animal research in Brazil. This includes: (i) permits from The Brazilian

547 Institute for the Environment and Renewable Natural Resources (IBAMA, no. 33094); (ii)

548 permission from The Brazilian Enterprise for Agricultural Research (EMBRAPA) at Sete Lagoas;

549 (iii) permission from landowners at the Divinópolis site to conduct the study on their property;

550 and (iv) tacit approval from the Brazilian Federal Government implied by employing authors to

551 conduct scientific research. None of the sampled species had protected status. No genetic

552 information was accessed in the study. 


\section{References}

Adams, E.S., Levings, S.C. (1987). Territory Size and Population Limits in Mangrove Termites. The Journal of Animal Ecology, 56, 1069-1081. https://doi.org/10.2307/4967

Akino, T., Knapp, J. J., Thomas, J. A., \& Elmes, G. W. (1999). Chemical mimicry and host specificity in the butterfly Maculinea rebeli, a social parasite of Myrmica ant colonies. Proceedings of the Royal Society B: Biological Sciences, 266(1427), 1419-1426. https://doi.org/10.1098/rspb.1999.0796

Altmann, J. (1974). Observational Study of Behaviour: Sampling Methods. Behaviour, 49, 227267. https://doi.org/10.1163/156853974X00534

Araujo, R.L. (1970). Termites of the Neotropical region. In: Biology of Termites (Ed. by K. Krishna \& F. M. Weesner), 527-576. Academic Press, London, U.K.

Aureli, F., Cords, M., van Schaik, C.P. (2002). Conflict resolution following aggression in gregarious animals: a predictive framework. Animal Behavior, 64, 325-343. https://dx.doi.org/10.1006/anbe.2002.3071

Baan, C., Bergmüller, R., Smith, D.W., Molnar, B. (2014). Conflict management in free-ranging wolves, Canis lupus. Animal Behaviour, 90, 327-334. https://dx.doi.org/10.1016/j.anbehav.2014.01.033

Binder, B. F. (1988). Intercolonial Aggression in the Subterranean Termite Heterotermes Aureus (Isoptera: Rhinotermitidae). Psyche: A Journal of Entomology, 95(1-2), 123-137. https://dx.doi.org/10.1155/1988/28452

Brandes, U., Erlebach, T. (2005). Network analysis: methodological foundations. Springer Science \& Business Media. https://doi.org/10.1007/b106453

Buschinger, A. (2009). Social parasitism among ants: a review (Hymenoptera: Formicidae). Myrmecological News, 12(3), 219-235.

Campbell, C., Russo, L., Marins, A., DeSouza, O., Schönrogge, K., Mortensen, D., Tooker, J., Albert, R., Shea, K. (2016). Top-down network analysis characterizes hidden termite-termite interactions. Ecology and Evolution, 6(17), 6178-6188. https://doi.org/10.1002/ece3.2313

Coaton, W. (1971). Five new termite genera from South West Africa. Cimbebasia 2, 1-33.

Collins, N. (1980). Inhabitation of epigeal termite (Isoptera) nests by secondary termites in Cameroun rain forest. Sociobiology 5, 47-53.

Costa, D.A., Carvalho, R.A. de, Lima-Filho, G.F. \& Brandão, D. (2009). Inquilines and invertebrate fauna associated with termite nests of Cornitermes cumulans (Isoptera, Termitidae) in the Emas National Park, Mineiros, Goias, Brazil. Sociobiology, 53, 443-454.

Crawley, M.J. (2012). The R Book. John Wiley \& Sons Ltd.

Cristaldo, P. F., Rosa, C. S., Florencio, D. F., Marins, A., \& DeSouza, O. (2012). Termitarium volume as a determinant of invasion by obligatory termitophiles and inquilines in the nests of Constrictotermes cyphergaster (Termitidae, Nasutitermitinae). Insectes sociaux, 59(4), 541548. https://doi.org/10.1007/s00040-012-0249-3

Cristaldo, P.F., DeSouza, O., Krasulová, J., Jirosova, A., Kutalová, K., Lima, E.R., Sobotnik, J., SillamDussès, D. (2014). Mutual use of trail-following chemical cues by a termite host and its inquiline. PLoS One, 9, https://doi.org/10.1371/journal.pone.0085315

Cristaldo, P.F., Jandák, V., Kutalová, K., Rodrigues, V.B., Brothánek, M., Jiříček, O., DeSouza, O., Šobotník, J. (2015). The nature of alarm communication in Constrictotermes cyphergaster (Blattodea: Termitoidea: Termitidae): the integration of chemical and vibroacoustic signals. Biology Open, 4, 1649-1659. https://doi.org/10.1242/bio.014084

Cristaldo, P.F., Rodrigues, V.B., Elliot, S.L., Araújo, A.P.A., DeSouza, O. (2016a). Heterospecific detection of host alarm cues by an inquiline termite species (Blattodea: Isoptera: Termitidae). Animal Behaviour, 120, 43-49. https://doi.org/10.1016/j.anbehav.2016.07.025

Cristaldo, P.F., Araújo, A.P.A., Almeida, C.S., Cruz, N.G., Ribeiro, E.J.M., Rocha, M.L., Santana, A.S., Santos, A.A., Oliveira, A.P., DeSouza, O., Florencio, D.F. (2016b). Resource availability influences aggression and response to chemical cues in the Neotropical termite 
Nasutitermes aff. coxipoensis (Termitidae: Nasutitermitinae). Behavioural Ecology and Sociobiology, 70, 1257-1265. https://doi.org/10.1007/s00265-016-2134-y

Cunha, H.F., Costa, D.A., Espírito-Santo Filho, K., Silva, L.O., Brandão, D. (2003). Relationship between Constrictotermes cyphergaster and Inquiline Termites in the Cerrado (Isoptera: Termitidae). Sociobiology 42, 761-770.

Darlington, J.P.E.C. (2011). Termites (Isoptera) as secondary occupants in mounds of Macrotermes michaelseni (Sjöstedt) in Kenya. Insectes Sociaux, 59, 159-165.

Dawkins, R., Krebs, J. R. (1979). Arms Races between and within Species. Proceedings of the Royal Society B: Biological Sciences, 205(1161), 489-511. https://doi.org/10.1098/rspb.1979.0081

Delaplane, K. (1991). Tests for intraspecific agonism in a Louisiana population of Coptotermes formosanus (Isoptera: Rhinotermitidae). J. Entomol. Sci. 26, 357-359.

Delphia, C. M., Copren, K. A., \& Haverty, M. I. (2003). Agonistic behavior between individual worker termites from three cuticular hydrocarbon phenotypes of Reticulitermes (Isoptera: Rhinotermitidae) from northern California. Annals of the Entomological Society of America, 96(4), 585-593. https://doi.org/10.1603/0013-8746(2003)096[0585:ABBIWT]2.0.C0;2

Deneubourg, J. L. (1977). Application de l'ordre par fluctuations a la description de certaines etapes de la construction du nid chez les termites. Insectes sociaux, 24(2), 117-130.

DeSouza, O., Miramontes, O., Santos, C. A., \& Bernardo, D. L. (2001). Social facilitation affecting tolerance to poisoning in termites (Insecta, Isoptera). Insectes Sociaux, 48(1), 2124. https://doi.org/10.1007/pl00001739

DeSouza, O., Araújo, A.P.A., Florencio, D.F., Rosa, C.S., Marins, A., Costa, D.A., Rodrigues, V.B., Cristaldo, P.F. (2016). Allometric scaling of patrolling rate and nest volume in Constrictotermes cyphergaster termites: hints on the settlement of inquilines. PLoS One,11(1), e0147594. https://doi.org/10.1371/journal.pone.0147594

De Visser, S.N., Freymann, B.P., Schnyder, H. (2008). Trophic interactions among invertebrates in termitaria in the African savanna: a stable isotope approach. Ecological Entomology, 33, 758-764.

Dias, P.A.D., Rangel-Negrín, A., Coyohua-Fuentes, A., Canales-Espinosa, D. (2009). Behaviour accumulation curves: a method to study the completeness of behavioural repertoires. Animal Behaviour, 77, 1551-1553. https://doi.org/10.1016/j.anbehav.2009.02.015

Eggleton, P., Bignell, D.E. (1997). Secondary occupation of epigeal termite (Isoptera) mounds by other termites in the MbalmayForestst Reserve, southern Cameroon, and its biological significance. Journal of African Zoology, 111, 489-498.

Eisner, T., Kriston, I., \& Aneshansley, D. J. (1976). Defensive behavior of a termite (Nasutitermes exitiosus)Behavioural Ecology and Sociobiology, 1(1), 83-125. https://doi.org/10.1007/BF00299954

Emerson, A. E. (1938). Termite nests: a study of the phylogenof behaviouror. Ecological Monographs, 8(2), 247-284.

Florane, C., Bland, J., Husseneder, C., Raina, A. (2004). Diet-mediated inter-colonial aggression in the Formosan subterranean termite Coptotermes formosanus. Journal of Chemical Ecology. 30, 2559-2574. https://doi.org/10.1007/s10886-004-7950-2

Florencio, D.F., Marins, A., Rosa, C.S., Cristaldo, P.F., Araújo, A.P.A., Silva, I.R., DeSouza, O. (2013). Diet segregatiobetween $\mathrm{n}$ cohabiting builder and inquiline termite species. PLoS One, 8 , e66535. https://doi.org/10.1371/journal.pone.0066535

Freeman, L.C. (1978). Centrality in social networks conceptual clarification. Social Networks, 1(3), 215-239. https://doi.org/10.1016/0378-8733(78)90021-7

Gobush, K.S., Wasser, S.K. (2009). Behavioural correlates of low relatedness in African elephant core groups of a poached population. Animal Behaviour, 78(5), 1079-1086. https://doi.org/10.1016/j.anbehav.2009.06.034

Gordon, D. M. The Ecology of Collective Behavior in Ants (2019) Annual Review of Entomology, 64:1, 35-50. https://doi.org/10.1146/annurev-ento-011118-111923

Grassé, P. P. (1986). Termitologia: Anatomie-Physiologie-Biologie-Systématique des Termites, Tome III Comportement-Socialité-Écologie-Évolution-Systématique. Paris Masson. 
Gravel, D., Guichard, F., Hochberg, M.E. (2011). Species coexistence in a variable world. Ecology Letters, 14, 828-839. https://doi.org/10.1111/j.1461-0248.2011.01643.x

Haverty, M.I., Thorne, B.L. (1989). Agonistic behaviour correlated with hydrocarbon phenotypes in dampwood termites, Zootermopsis (Isoptera: Termopsidae). Journal of Insect Behaviour. 2, 523-543. https://doi.org/10.1007/BF01053352

Hölldobler, B., Wilson, E.O. (1990). ThAntsts. Harvard University Press.

Ishikawa, Y., Miura, T. (2012). Hidden aggression in termite workers: plastic defensive behaviour dependent upon social context. Animal Behaviour, 83, 737-745. https://doi.org/10.1016/j.anbehav.2011.12.022

Jirošová, A., Sillam-Dussès, D., Kyjaková, P., Kalinová, B., Dolejšová, K., Jančařík, A., Majer, P., Cristaldo, P.F., Hanus, R. (2016). Smells like home: chemically mediated co-habitation of two termite species in a single nest. Journal Chemical Ecology, 42, 1070-1081. https://doi.org/10.1007/s10886-016-0756-1

Kays, R., Crofoot, M. C., Jetz, W., Wikelski, M. (2015). Terrestrial animal tracking as an eye on life and planet. Science, 348(6240), aaa2478. https://doi.org/10.1126/science.aaa2478

Kilner, R., Langmore, N. (2011). Cuckoos versus hosts in insects and birds: adaptations, counteradaptations and outcomes. Biol. Rev. 86, 836:852.

Kistner, D. (1969). The biology of termitophiles. Biology of Termites, Vol. 1 (ed. by K. Krishna and F. M. Weesner), pp. 525 - 557. Academic Press, London, U.K.

Kistner, D. (1979). Social and evolutionary significance of social insect symbion.sI in: Hermann, H. (Ed.), Social Insects. Academic Press, New York, pp. 339-413.

Kistner, D. (1990). The integration of foreign insects into termite societies or why do termites tolerate foreign insects in their societies? Sociobiology, 17, 191-215.

Korb, J., Linsenmair, K.E. (1999). Reproductive success of Macrotennes bellicosus (Isoptera, Macroteritinae) in two neighbouring habitats. Oecologia 118, 183-191.

Korb, J., Buschmann, M., Schafberg, S., Liebig, J., Bagnères, A. G. (2012). Brood care and social evolution in termites. Proceedings. BiologicaScienceses, 279(1738), 2662-71.

Kottek, M., Grieser, J., Beck, C., Rudolf, B., Rubel, F. (2006). World map of the Köppen-Geiger climate classification updated. Meteorologische Zeitschrift, 15(3), 259-263. https://doi.org/10.1127/0941-2948/2006/0130

Krishna, K., Grimaldi, D., Krishna, V., Engel, M.S. (2013). Treatise on the Isoptera of the World: Nasutitermitinae. In: Bulletin of the American Museum of Natural History, 377(5),14991901.

Lehner, P.N. (1998). Handbook of ethological methods. Cambridge Univ Press.

Levings, S.C., Adams, E.S. (1984). Intra- and interspecific territoriality in Nasutitermes (Isoptera: Termitidae) in a Panamanian Mangrove forest. The Journal of Animal Ecology, 53, 705-714. https://doi.org/10.2307/4653

Lhomme, P., Ayasse, M., Valterová, I., Lecocq, T., \& Rasmont, P. (2012). Born in an Alien Nest : How Do Social Parasite Male Offspring Escape from Host Aggression? PLoS One, 7(9), e43053. https://doi.org/10.1371/journal.pone.0043053

Marins, A.; Costa, D.; Russo, L.; Campbell, C.; DeSouza, O.; Bjønrstad, O., Shea, K. (2016). Termite cohabitation: the relative effect of biotic and abiotic factors on mound biodiversity. Ecological Entomology, 41, 532-541

Mathews, A.G.A. (1977). Studies on termites from the Mato Grosso State, Brazil (p 267). Rio de Janeiro, Academia Brasileira de Ciências.

Matsuura, K. (2010). Sexual and Asexual Reproduction in Termites. Biology of Termites: a Modern Synthesis, 255-277. https://doi.org/10.1007/978-90-481-3977-4_10

Miramontes, O., DeSouza, O. (1996). The Nonlinear Dynamics of Survival and Social Facilitation in Termites. Journal of Theoretical Biology, 181(4), 373380. https://doi.org/10.1006/jtbi.1996.0138

Miramontes, O., DeSouza, O. (2008). Individual basis for collective behaviour in the termite, Cornitermes cumulans. Journal of Insect Science, 8, 24.

Miura, T., Matsumoto, T. (1997). Open-air litter foraging in the nasute termite Longipeditermes longipes (Isoptera: Termitidae). Journal of Insect Behavior, 11, 179-189. 
Monteiro, I., Viana-Junior, A. B., de Castro Solar, R. R., de Siqueira Neves, F., DeSouza, O. (2017). Disturbance-modulated symbioses in termitophily. Ecology and Evolution, 7(24), 1082910838. https://doi.org/10.1002/ece3.3601

Moura, F.M.S., Vasconcellos, A., Araújo, V.F.P., Bandeira, A.G. (2006). Seasonality in foraging behaviour of Constrictotermes cyphergaster (Termitidae, Nasutitermitinae) in the Caatinga of Northeastern Brazil. Insectes Sociaux, 53, 472-479. https://doi.org/10.1007/s00040005-0899-0

Nash, D., Boomsma, J. (2008). Communication between hosts and social parasite. In: D'Ettorre, P., Hughes, D. (Eds.), Sociobiology of communication: an interdisciplinary perspective. Oxford University Press, 55-79.

Oulhen, N., Schulz, B.J., Carrier, T.J. (2016). English translation of Heinrich Anton de Bary's 1878 speech, 'Die Erscheinung der Symbiose' ('De la symbiose'). Symbiosis, 69, 131-139. https://doi.org/10.1007/s13199-016-0409-8

Nehring, V., Dani, F.R., Turillazzi, S., Boomsma, J.J., d'Ettorre, P. (2015). Integration strategies of a leaf-cutting ant social parasite. Animal Behaviour 108, 55-65. https://doi.org/10.1016/j.anbehav.2015.07.009

Neoh, K.-B., Indiran, Y., Lenz, M., Lee, C.-Y. (2012). Does lack of intraspecific aggression or absence of nymphs determine acceptance of foreign reproductives in Macrotermes? Insectes Sociaux, 59, 223-230. https://doi.org/10.1007/s00040-011-0207-5

Noirot, C. (1970). The nests otermitesit. in: Krishna, K., Weesner, F.M. (Eds.), Biology of Termites. Academic Press, 73-125.

Pierce, N.E., Braby, M.F., Heath, A., Lohman, D.J., Mathew, J., Rand, D.B., Travassos, M.A. (2002). The ecology and evolution of ant association in the Lycaenidae (Lepidoptera). Annual Review of Entomology, 47(1), 733-771. https://doi.org/10.1146/annurev.ento.47.091201.145257

R Development Core Team (2018) R: A Language and Environment for Statistical Computing. $R$ Foundation for Statistical Computing, Vienna, Austria.

Ratter, J., Ribeiro, J., Bridgewater, S. (1997). The Brazilian Cerrado Vegetation and Threats to its Biodiversity. Annals of Botany, 80(3), 223-230. https://doi.org/10.1006/anbo.1997.0469

Redford, K. H. (1984). The Termitaria of Cornitermes cumulans (Isoptera, Termitidae) and Their Role in Determining a Potential Keystone Species. Biotropica, 16(2), 112. https://doi.org/10.2307/2387842

Rodrigues, V. B., Costa, D. A., Cristaldo, P. F., DeSouza, O. (2018). Lagged population growth in a termite host colony: cause or consequence of inquilinism? Neotropical Entomology. https://doi.org/10.1007/s13744-018-0634-3

Roisin, Y., Everaerts, C., Pasteels, J.M., Bonnard, O. (1990). Caste-dependent reactions to soldier defensive secretion and chiral alarm/recruitment pheromone in Nasutitermes princeps. Journal of Chemical Ecology, 16, 2865-2875. https://doi.org/10.1007/BF00979479

Shellman-Reeve, J. (1997). The spectrum of eusociality in termet. I in: The evolution of social behaviour in insects and arachnids, pp. 52-93, Cambridge University Press.

Stuart, A. M. (1967). Alarm, defense, and construction behavior relationships in termites (Isoptera). Science, 156(3778), 11231125https://doi.org/i:10.1126/science.156.3778.1123

Su, N.-Y., Haverty, M. I. (1991). Agonistic behavior among colonies of the Formosan subterranean termite, Coptotermes formosanus Shiraki (Isoptera: Rhinotermitidae), from Florida and Hawaii: Lack of correlation with cuticular hydrocarbon composition. Journal of Insect Behavior, 4(1), 115-128. https://doi.org/10.1007/BF01092555

Sumpter, D. J. T. (2006). The principles of collective animal behaviour. Philosophical Transactions of the Royal Society B: Biological Sciences, 361(1465), 5-22. https://doi.org/10.1098/rstb.2005.1733

Thierry, B., Aureli, F., Nunn, C. L., Petit, O., Abegg, C., De Waal, F. B. M. (2008). A comparative study of conflict resolution in macaques: insights into the nature of trait covariation. Animal Behaviour, 75(3), 847-860. http://dx.doi.org/10.1016/j.anbehav.2007.07.006 
768 Tokeshi, M. (2009). Species Coexistence: ecological and evolutionary perspectives. Wiley Online 769 Library.

770 Vasconcellos, A., Araújo, V., Moura, F., Bandeira, A. (2007). Biomass and Population Structure of Constrictotermes cyphergaster (Silvestri) (Isoptera: Termitidae) in the dry forest of caatinga, Northeastern Brazil. Neotropical Entomology, 36, 693-698. http://dx.doi.org/10.1590/S1519-566X2007000500009

Wilson, E. (1988). The current state of biological diversity. Biodiversity, 521, 3-18, Washington DC, The National Academies Press. https://doi.org/10.17226/9 
bioRxiv preprint doi: https://doi.org/10.1101/587592; this version posted March 24, 2019. The copyright holder for this preprint (which was

not certified by peer review) is the author/funder, who has granted bioRxiv a license to display the preprint in perpetuity. It is made 